\title{
Features of technological basing in machining parts on CNC machines
}

\author{
Nicolay V. Nosov ${ }^{1, *}$, and Andrey A. Cherepashkov ${ }^{1}$ \\ ${ }^{1}$ Samara State Technical University (SamSTU ), Mechanical Engineering Technology Dep., RF
}

\begin{abstract}
The article discusses the problems of computer aided design and technological preparation of production of complex shape parts on machines with numerical program control. An integrated technique for designing processes and control programs for $\mathrm{CNC}$ machines is proposed and described, using the technique of software-based referencing and modern electronic measuring tools.
\end{abstract}

\section{Introduction}

Computer aided design and technological preparation of production (CAD / CAM / CAE ... - CA"x"-technologies) are among the most important methods and means of information support for modern machine building [1]. Moreover, as practice shows, the greatest effect is achieved with the integrated application of technologies and technical means of industrial automation [2].

For industrial enterprises, the issues of automation of the design and production stages of the product life cycle and the corresponding basic CAD technologies: computer-aided geometric modeling and design (CAD), design of control programs for CNC machine tools (CAM), as well as automated measurement of parts form (CAI) are the most pressing ones. Practice shows that the above mentioned design procedures and processes are substantially interdependent [3]. As early as at the stage of geometric modeling of the basing surfaces of the parts being machined it is necessary to take into account the accuracy, tools, and equipment involved in manufacturing. In their turn, all the above-listed issues must be taken into account and specified in the selection of processing strategies and parameters of control programs. The complex character of computerization of design and technological preparation of production, manufacturing and measurement of parts significantly changes the approaches to workpiece referencing on machining equipment.

The ideas of adaptive control of metal-working machinery based on direct measurement during cutting [4] and the use of geometric modeling of machined surfaces [5] are not new. But only at the present stage of the development of means of industrial automation and the introduction of technologies for their integrated use, these approaches (we will call them the "software" method of workpiece referencing) become practically achievable and economically feasible.

\footnotetext{
*Corresponding author: nosov.nv@samgtu.ru
} 


\section{The problem of automating the basing of parts in the complex automation of technological preparation of production}

The geometric model of the part, created by a designer in the environment of machinebuilding $\mathrm{CAD}$ is transformed in a certain way by a process engineer in the models of blanks, developed in the same CAD. But, it can be argued that modern methods of design automation substantially change the traditional approaches to the design of workpiece components and the development of technological processes.

As a rule, the dimensions of the blanks are included in the technological dimension chain from which the coordinate system is derived, using the main and auxiliary bases (surfaces). At the same time, the workpiece is attached to the details of the technological system by aligning its processing bases with the operative surfaces of the machining workstation or fixture. Because of this, the coordinate system changes its position and is constructed on the operating surfaces of the device or machine. Aligning the coordinate systems in this way, we obtain a set of coordinate systems connected with each other. But, since each component of the technological system is deprived of the relevant number of degrees of freedom, certain constraints must be imposed on the forming coordinate system. The role of constraints during machining is performed by reference points on the surface of the workpiece or tool. Thus, the displacement of reference points can be represented as a function of the properties of the constraints and the operating technological factors.

We set down the equation of motion in such a way that it includes factors that cause a violation of the trajectory of the relative motion of the tool and workpiece. For this, each displacement and rotation of each coordinate system is to be replaced by the displacement functions of their reference points. And the displacement of reference points is, in turn, represented as a function of properties, relationships and operating factors.

As a result, the equation of coordinates of the points of the cutting edges (given in the coordinate system of the tool) in the coordinate system constructed on the technological bases of the part, that is,

$$
\begin{aligned}
& x_{d}=f_{1}\left(x_{c}, y_{c}, z_{c}, t\right), \\
& y_{d}=f_{2}\left(x_{c}, y_{c}, z_{c}, t\right), \\
& z_{d}=f_{3}\left(x_{c}, y_{c}, z_{c}, t\right),
\end{aligned}
$$

where $x_{d}, y_{d}, z_{d}$ are the coordinates of the point $M$ of the tool cutting edges in the part coordinate system, $x_{c}, y_{c}, z_{c}$ are the coordinates of the point $M$ of the tool cutting edges in the tool coordinate system, $t$ is the motion parameter.

Consider the position of the point $M$ of the cutting edge of the tool when changing from one coordinate system to another. The position of the point $M$ is established for a successive transition through all coordinate systems of an equivalent scheme to the coordinate system of the part.

The equation of motion of the point $M$ is obtained which contains the radius vectors $\left(r_{i}\right)$ of the origin of the coordinate systems and the matrices $\left(M_{i}\right)$ of three successive rotations of each coordinate system, that is,

$$
\bar{R}=f\left(\bar{r}_{1}, \bar{r}_{2}, \ldots, \bar{r}_{n}, M_{1}, M_{2}, \ldots, M_{n}\right)
$$

where $n$ is the number of coordinate systems of the equivalent scheme of the technological system.

In its turn 


$$
\bar{r}_{i}=\left\|\begin{array}{c}
x_{i} \\
y_{i} \\
z_{i}
\end{array}\right\| ; \quad M_{i}=M_{i z} \circ M_{i y} \circ M_{i x},
$$

where $x_{i}, y_{i}, z_{i}$ are the coordinates of the origin (point) of the $i$-th coordinate system, $M_{i z}, M_{i y}, M_{i x}$ - matrices of rotations of the $i$-th coordinate system around the axes $O Z, O Y, O X$.

Wherein

$$
\begin{aligned}
M_{i x} & =\left\|\begin{array}{ccc}
1 & 0 & 0 \\
0 & \cos \varphi & -\sin \varphi \\
0 & \sin \varphi & \cos \varphi
\end{array}\right\| ; \\
M_{i y} & =\left\|\begin{array}{ccc}
\cos \omega & 0 & \sin \omega \\
0 & 1 & 0 \\
-\sin \omega & 0 & \cos \omega
\end{array}\right\| ; \\
M_{i z} & =\left\|\begin{array}{ccc}
\cos \theta & \sin \theta & 0 \\
\sin \theta & -\cos \theta & 0 \\
0 & 0 & 1
\end{array}\right\|,
\end{aligned}
$$

where $\theta, \omega, \varphi$ are the angles of rotations of the coordinate systems, around the axes $O X, O Y, O Z$ respectively.

Thus, the equation of motion of the point $M$ will contain six parameters that determine the positions of each coordinate system in the equivalent system.

Mathematically we describe the relationship between the operating factors and the total error of machining through the sequential establishment of functional links. This system establishes the links between the operating factors and the displacements of the reference points, the displacements of the reference points and the six position parameters of each coordinate system, the positions of the coordinate systems and the radius vector of a point of the surface of the machined part.

Determining the error of machining at each point of the surface, we find the error of the entire machined surface of the part. This will make it possible, with the help of the known techniques, to determine the accuracy of the part in terms of size, shape and location as applied to relative rotations and the geometric shape of its surfaces.

The method of determining errors in the installation of the workpiece, static and dynamic adjustment of the technological system does not fundamentally differ from the procedure for determining the total error of machining. The only difference is in equivalent schemes.

The construction of an equivalent scheme for calculating errors at each stage begins with the detection of dimension chains of the installation, static and dynamic settings. The difference between dimension chains determines the difference between equivalent schemes and the form of the equation of relative motion. 


\section{Implementation of the methodology of software-based referencing}

As follows from the above, the main stages in the construction of a mathematical model are: choosing the accuracy index, constructing an equivalent scheme, setting down the equation of relative motion, and introducing operative factors and qualitative characteristics into the equation of motion.

With the help of this kind of mathematical models and basic tools of complex design automation systems, a wide range of tasks related to the calculation, provision and prediction of the accuracy of machines can be solved both at the stage of their design, manufacture, and during operation.

It is not always possible to coordinate technological systems (billets, devices and machine tools), especially in the case of universal machines. When CNC machines are used for machining parts this coordination can be carried out using the method of software-based referencing.

The coordination of the processes of geometric modeling of a box-shaped workpiece (Fig. 1) with the design and technological bases contributes to the achievement of an optimal result in the manufacture, which ensures an improvement in the quality of the products manufactured and the productivity of the process.

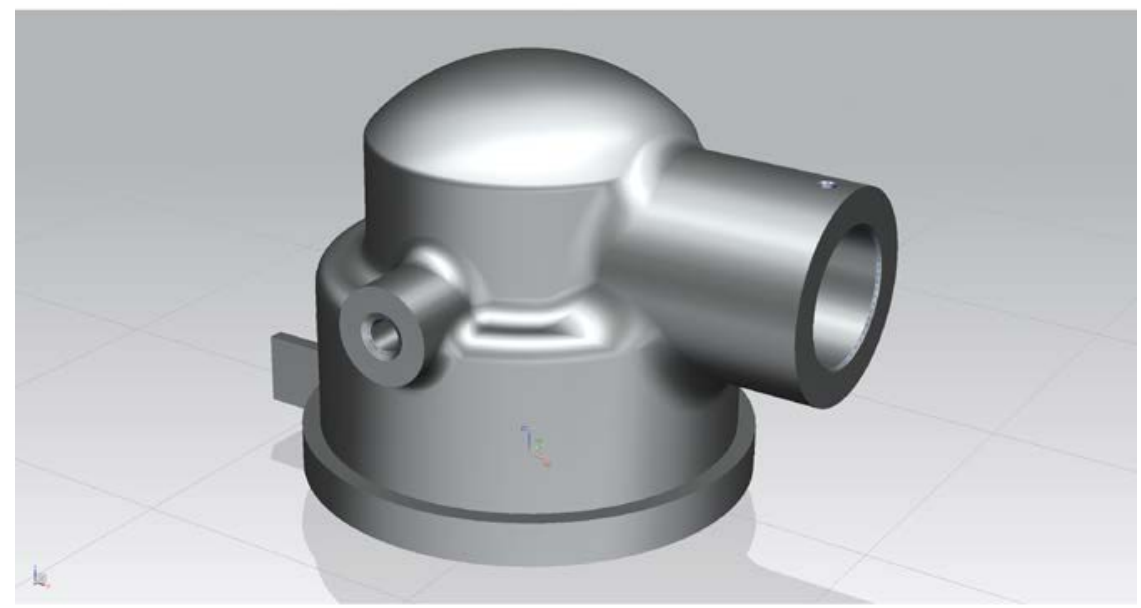

Fig. 1. 3D model of box-shaped workpiece

As a rule, the machining of box-shaped workpieces begins with the preparation of technological bases that are used subsequently. In the traditional machining method (Fig.2, a), the basic design surfaces (plane and cylindrical surface) are used as technological bases, while auxiliary design surfaces are machined.

Unlike traditional methods of billet referencing (machining of workpieces on universal and unit-type machine tools), the method of software-based referencing can be implemented on $\mathrm{CNC}$ machines equipped with software-controlled electronic measuring instruments. In this case, the development of control programs containing measurement operations and appropriate adjustment of coordinate systems allows simultaneous treatment of the main and auxiliary design surfaces (Fig. 2, b). 


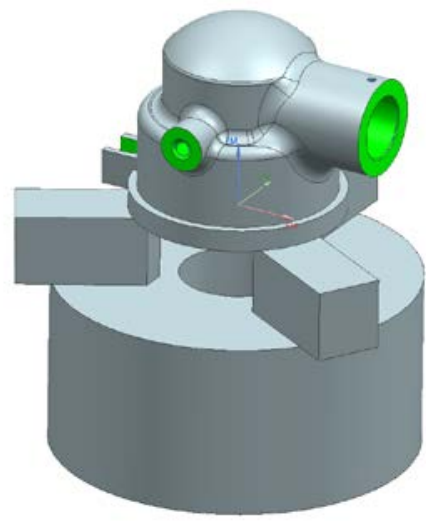

a

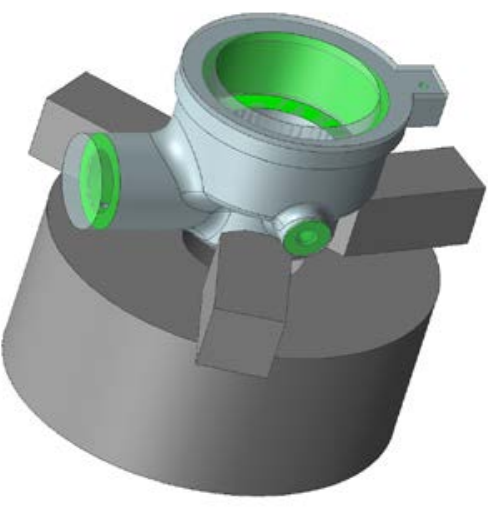

b

Fig. 2. Installation of workpiece machined according to the traditional scheme of referencing (a) and software-based referencing (b)

\section{General results and conclusions}

Software-based referencing differs from the "manual" methods in the fact that by means of programming the inverse task is solved - the correction of the position of the main machining axes of a CNC machine relative to the coordinates of the base points measured on the surface of the workpiece fixed on the machining workstation.

When using software-based referencing, it becomes possible to install the workpiece in the fixture on the table easily and quickly though relatively coarsely. Possibilities of multiaxis working (3-6 axes) and presence of built-in digital measuring instruments are necessary conditions for the implementation of this method on CNC machines.

When using software-based referencing, from the point of view of the process engineer, the development of the technological process combines the resources of design, technological and measuring bases.

The combination of machining and measurements in one program makes it possible to significantly reduce the overall duration of the manufacturing cycle, and, most importantly, dramatically improve the accuracy of maintaining dimensions and tolerances.

\section{References}

1. A.A. Cherepashkov, N.V. Nosov, Computer technologies, modeling and automated systems in machine building (Prospekt Nauki, St. Petersburg, 2018).

2. A.A. Cherepashkov, Izvestia of Samara Scientific Center of the Russian Academy of Sciences, v. 12(33), pp. 619-622 (2010).

3. K.V. Khrustitskiy, A.A. Cherepashkov, Bulletin of the Samara State Aerospace University, v. 5 (36), pp. 61 - 71 (2012).

4. B. S. Balakshin, Adaptive management of the tool (Machine-building, Moscow, 1973).

5. B.M. Bazrov, Module technology in machine building (Machine-building, Moscow, 2001). 